\title{
Computed tomography with normal chest radiograph in tuberculous infection
}

Christophe Delacourt, Tania Mamou Mani, Valérie Bonnerot, Jacques de Blic, Natacha Sayeg, Denis Lallemand, Pierre Scheinmann

\begin{abstract}
Children with primary tuberculosis infection without disease must be identified and treated preventively to avoid an increase in the incidence of tuberculosis in children. However, the recognition of infected cases without disease is often difficult. In particular, minimal active disease may be present in many cases but unrecognised on chest radiography. Computed tomography was therefore performed in 15 children with tuberculous infection and a normal chest radiograph to measure the size of their mediastinal lymph nodes. Ten control children without tuberculosis were also evaluated. When compared with controls it was found that nine of $15(60 \%)$ infected children had enlarged lymph nodes. Adenopathies were more frequent in infected children less than 4 years old than in those over 8 years old. The demonstration of unrecognised active disease in many infected children raises the question of the adequate treatment for these children. It is proposed that a two drug regimen would be more appropriate than isoniazid alone in these cases.

(Arch Dis Child 1993; 69: 430-432)
\end{abstract}

Despite the numerous efforts to control tuberculosis in developed countries, the disease remains a major health problem especially in urban areas. The growing number of new cases in adults naturally represents a source of infection for children. In Paris, the number of cases of new active disease generally decreased each year until 1986 . However, this decades long decline has ceased: the incidence in 1989 was $57 / 100000$ contrasting with a national incidence of $16 / 100000 .^{1}$ Furthermore, children with primary tuberculous infection are the reservoir from which future cases will emerge. ${ }^{2}$ Present approaches must therefore continue to aim at adequate treatment of all cases of active disease, prevention of new cases of tuberculosis by treating infected patients without disease, and prevention of infection of those who are not currently infected. ${ }^{3}$

One of the major practical problems in diagnosing tuberculosis in children is distinguishing recent infection without disease from infection with disease. Given a history of exposure to active pulmonary disease and a positive tuberculin skin test, the chest radiograph findings would determine whether the child receives only preventive treatment or curative treatment. However, the interpretation of the chest radiograph may be subtle and thus may mislead the practitioner. We therefore postulated that minimal active disease may be present but unrecognised from the chest radiograph in many children considered as having only tuberculous infection without disease. In this study, we performed computed tomography in children with tuberculous infection and a normal chest radiograph to assess the presence of enlarged lymph nodes and thus to confirm our hypothesis.

\section{Patients and methods}

CHILDREN

Fifteen children who satisfied the criteria for tuberculous infection without disease were included in this study. Nine were less than 4 years old with a mean (SD) age of $2.0(0.9)$ years (range $0.6-3.3$ ) and six were more than 8 years old, with a mean age of $10.6(2.0)$ years $(8 \cdot 0-13 \cdot 8)$. Of these 15 children, seven originated from European countries and eight came from developing countries. Ten had previously been vaccinated with BCG. Diagnostic criteria were as follows: (1) Recent exposure to active pulmonary disease and increase of $10 \mathrm{~mm}$ or more of their intradermal skin test reaction size $(n=6)$, or no known contact with active disease, but recent conversion of their intradermal skin test (a positive result $>15 \mathrm{~mm}$ in diameter) $(n=9)$. (2) Normal clinical examination. (3) Normal chest radiograph. The normality was assessed by three separate investigators, one paediatrician from our unit and two radiologists. (4) Three negative gastric aspirates.

As normal sizes of mediastinal lymph nodes in children have not been defined, 10 age matched control children were also included. Five were less than 4 years old with a mean age of $1.5(0.6)$ years and five were more than 8 years old with a mean age of $10.6(1.5)$ years. Computed tomography was performed in these children for mediastinal lipoma $(n=1)$, congenital cardiopathy $(n=4)$, or screening for metastasis of bone tumour $(n=5)$. None of these children had history of bronchopulmonary infection during the preceding six weeks.

\section{COMPUTED TOMOGRAPHY}

All children with tuberculosis had computed tomograms of the chest before starting treatment. Contiguous sections from the apex to the diaphragm were obtained after intravenous bolus injection of contrast material $(2 \mathrm{ml} / \mathrm{kg}$ of weight). Fourteen of the 15 examinations were performed using an ultrafast scanner (Picker IMATRON C-100). Characteristics were: scan time $0 \cdot 1$ second, interscan delay 1 second, 


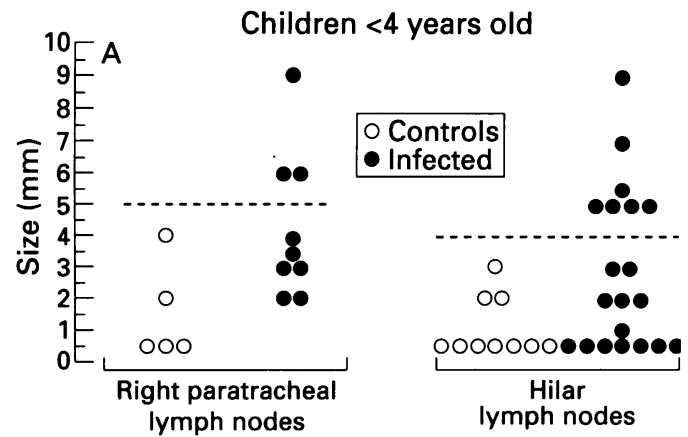

Children $>8$ years old
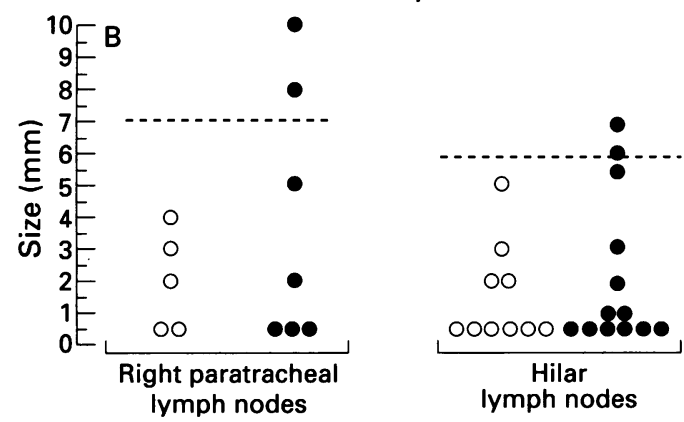

Figure 1 Size of lymph nodes in control and infected children at $(A)<4$ and $(B)>8$ years old; dashed line indicates cut off points defined in control children.

and section thickness $6 \mathrm{~mm}$. One 11 year old child was examined with a conventional scanner (GE 9800 Milwaukee) and the characteristics were 2 seconds, $10-20$ seconds, and $10 \mathrm{~mm}$, respectively. Of the control children, six underwent ultrafast computed tomography and four, more than 8 years of age, had conventional computed tomography. All mediastinal lymph nodes were examined and the anteroposterior axis measured.

\section{Results}

CONTROL CHILDREN

In control children, lymph nodes could be measured only in the right pretracheal and hilar positions, and were small. No lymph nodes were found in other sites. From these measurements, cut off points could be defined. In the right pretracheal position, lymph nodes were defined as enlarged if $\geqslant 5 \mathrm{~mm}$ in children $<4$ years old or if $\geqslant 7 \mathrm{~mm}$ in children $>8$ years old. In hilar zones, the cut off points were $\geqslant 4 \mathrm{~mm}$ and $\geqslant 6 \mathrm{~mm}$ respectively.

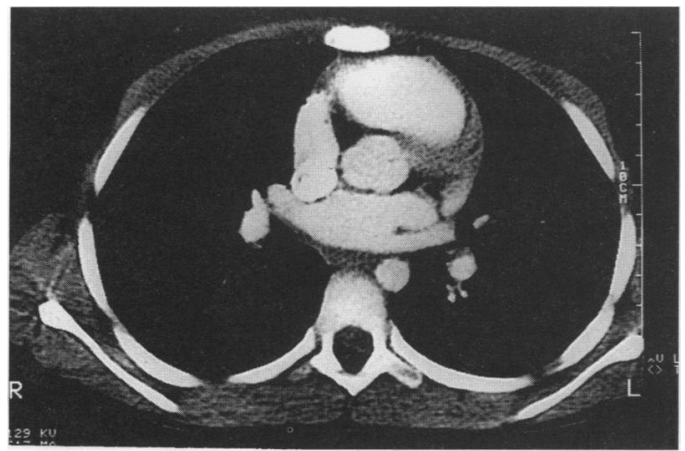

Figure 2 Infected child with normal size lymph nodes.
INFECTED CHILDREN

Many lymph nodes both in the right pretracheal and hilar positions in children with tuberculous infection were enlarged as compared with the control patients (fig 1). No adenopathy was found in other zones.

Six of the 15 infected children had no enlarged lymph node in any zone (fig 2 ). Three of these six children originated from Europe and recent contact with active tuberculosis was identified in three cases.

Six of the nine children $<4$ years old $(67 \%)$ had adenopathies in at least one zone. Two of these six children had two zones involved (fig 3). Four of these six children originated from Europe and the source of infection was identified in only three cases. The mean diameter of the enlarged lymph nodes was $7.0 \mathrm{~mm}$ in the right pretracheal zone and $5.9 \mathrm{~mm}$ in hilar zones. In children $>8$ years old, three of the six children had adenopathies in one zone. None had two zones involved. Two of these three children originated from Europe and none had recently been exposed to active tuberculosis. The mean diameter of enlarged lymph nodes was $9.0 \mathrm{~mm}$ in the right pretracheal zone and $6.5 \mathrm{~mm}$ in hilar zones.

\section{Discussion}

We have shown that enlarged lymph nodes may be detected by computed tomography in $60 \%$ of children with tuberculous infection and normal findings on chest radiography.

Children with primary tuberculosis infection are the reservoir from which future cases will emerge. To prevent an increase in the incidence of pulmonary and extrapulmonary forms of tuberculosis in children, it is necessary to identify and preventively to treat infected cases without disease. The success of preventive treatment programmes depends on the recognition of infected children without disease and on the use of an effective and well tolerated drug regimen.

Recognition of asymptomatic infected patients may be difficult in paediatric practice. The absence of radiographic evidence of tuberculosis usually leads to the conclusion that there is no disease. ${ }^{4}$ However, our study shows that more than half of the infected children with normal chest radiography had enlarged lymph nodes identified by computed tomography. Thus, a normal chest radiograph may

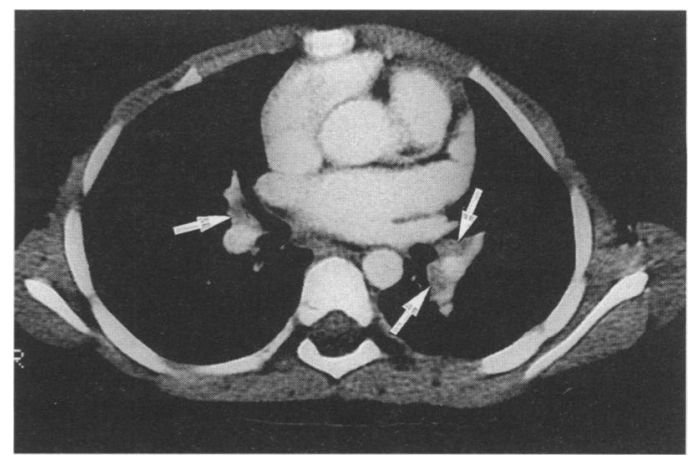

Figure 3 Infected child with enlarged lymph nodes in two zones. 
often correspond to minimal active disease rather than to the absence of disease. In the pathogenesis of tuberculosis, hilar and mediastinal lymph nodes are always seeded with bacilli during the initial infection, ${ }^{4}$ and our results support the conclusion that infection of these nodes progresses to active disease more often than suggested by chest radiograph findings. This is supported by our group of infected children under 4 years of age, of which two thirds had adenopathies. This correlates with the known, greater risk of developing severe disease in young children. ${ }^{5}$

Current guidelines for treatment of children with tuberculosis state that having enlarged nodes in the chest means that treatment with two drugs should last for six months. If there is infection without disease, treatment is either three months with two drugs or six months with one. ${ }^{6}$ Our findings of minimal active disease in infected children with normal chest radiography raises the question of adequate therapeutic management in these children. The aim of our study was not to change current guidelines for treatment, but our results suggest that further thought needs to be given to the wisdom of dividing patients into two treatment categories based on the appearance of the chest radiograph. This problem is of importance as about three quarters of children with tuberculous infection have normal findings on chest radiography. ${ }^{7}$ Furthermore, when tuberculin positive children are found to be free of tuberculous disease, there is controversy about the value of chemoprophylaxis. $^{8}$ The decision to treat is influenced by contact, ethnic origin, and BCG history. ${ }^{8}$ Primary tuberculosis usually tends to heal, ${ }^{9}$ and the morbidity rate of untreated tuberculous infection with normal initial chest radiography was estimated to $10 \cdot 1$ per $1000 .^{10}$ Furthermore, preventive treatment in infected children with normal chest radiography reduces the risk of disease. ${ }^{11}$ However, although a six to 12 month course of isoniazid reduces morbidity from tuberculosis by $50 \%$ to $80 \%,{ }^{12}$ prophylactic treatment sometimes fails and the overt disease develops. ${ }^{13}$ Primary resistance to isoniazid and poor compliance may explain many of these failures. In a recent study, however, Glassroth et al showed that failure of isoniazid preventive treatment may at least partly account for up to $6 \%$ of new cases of active tuberculosis and that neither drug resistance nor poor compliance appeared to explain the failure of prevention in these cases. ${ }^{14}$ We speculate that progression of initially unrecognised clinically active disease could at least partly be responsible for the failure of a one drug preventive regimen, which would leave sensitive but persistent bacilli. ${ }^{15}$ A large trial would be required definitely to prove this hypothesis. The introduction of chemoprophylaxis with rifampicin and isoniazid seems therefore more appropriate than the use of isoniazid alone. Combination treatment aims at rapid killing of all populations of organisms to sterilise the lesions as quickly as possible. ${ }^{25}$ Prompt and vigorous treatment is especially important in children under 5 years of age. ${ }^{5}$ This two drug prophylactic treatment has given excellent results and is well tolerated in children. ${ }^{16}$

Images of enlarged lymph nodes in our population have been obtained with an ultrafast computed tomographic scanner. Their high quality permitted us a reliable measure of lymph node anteroposterior axes. In young children who are not able to hold their breath, this quality of chest computed tomogram is almost impossible to obtain with a conventional scanner, which therefore does not appear appropriate in investigating this problem. Thus, the purpose of our study is not to recommend that all tuberculin positive children with normal chest radiography should undergo computed tomography in order to distinguish children who might receive two drug chemoprophylaxis from those who might receive a single drug. Ultrafast computed tomographic scanning would not be available in most cases and the cost very high. We suggest that, given the evidence of the frequency of enlarged lymph nodes unrecognised on chest radiography, a dual drug regimen should be preferred in all infected children with normal chest radiography. Good tolerance and low cost argue for this option.

In summary, this study demonstrates that many infected children with normal chest radiography have unrecognised active disease. Two drug chemoprophylaxis would be more appropriate than isoniazid alone in these cases.

1 Vacarie M, Gambier J, Hubert B. La tuberculose à Paris en 1989. Bulletin Epidémiologique Hebdomadaire 1990: 89-91.

2 Starke JR. Modern approach of the diagnosis and treatment of tuberculosis in children. Pediatr Clin North Am 1988; 35: 441-64.

3 Bass JB Jr. Tuberculin test, preventive therapy and elimination of tuberculosis. Am Rev Respir Dis 1990;141: 812-3. 4 American Thoracic Society/Centers for Disease Control. Diagnostic standards and classification of tuberculosis. Am Rev Respir Dis 1990; 142: 725-35.

5 American Thoracic Society. Treatment of tuberculosis and tuberculosis infection in adults and children. Am Rev Respir Dis 1986; 134: 355-63.

6 Ormerod LP. Chemotherapy and management of tuberculosis in the United Kingdom: recommendations of the Joint Committee of the British Thoracic Society. Thorax $1990 ; 45$ : 403-8.

7 Inselman LS, El-Maraghy NB, Evans HE. Apparent resurgence of tuberculosis in urban children. Pediatrics 1981; 68: 647-9.

8 Citron K. Tuberculin positive children. Thorax 1992; 47: 768-9.

9 Inselman LS, Kendig EL, Jr. Tuberculosis. In: Chernick V, Kendig EL, eds. Disorders of the respiratory tract in children 5th Ed. Philadelphia: WB Saunders, 1990: 730-69.

10 Ferebee SH. Controlled chemoprophylaxis trials in tuberculosis: a general review. Advances in Tuberculosis Research 1969; 17: 28-106.

11 Geiter LJ, O'Brien RJ. Conference on new approaches for tuberculosis preventive therapy. $\mathcal{F}$ Infect Dis 1987; 156: 536-7.

12 International Union Against Tuberculosis Committee on Prophylaxis. Efficacy of various durations of isoniazid preventive therapy for tuberculosis: 5 years of follow-up in the IUAT trial. Bull World Health Organ 1982; 60: 555-64.

13 Hsu KHK. Thirty years after isoniazid. Its impact on tuberculosis in children and adolescents. $\mathscr{F} A M A$ 1984; 251 : culosis in $1283-5$.

14 Glassroth J, Bailey WC, Hopewell PC, Schecter G, Harden JW. Why tuberculosis is not prevented. Am Rev Respir Dis 1990; 141: 1236-40.

15 Reis FCJ, Bedran MBM, Moura JAR, Assis I, Rodrigues MESM. Six-month isoniazid-rifampicin treatment for pulmonary tuberculosis in children. Am Rev Respir Dis pulmonary tuberculo

16 Ormerod LP. Reduced incidence of tuberculosis by prophylactic chemotherapy in subjects showing strong reactions to tuberculin testing. Arch Dis Child 1987; 62: 1005-8. 